bodies. In other words, if the molecular energy of a socalled element could be changed, the element would be dissociated, a view of special interest in relation to the researches of Lockyer. The lecture was illustrated by many effective experiments, and concluded with the statement that it had not been instituted from the merely special interest of Graham's researches to the physicist and chemist, but in honour of the labours of a life the memory of which will be as enduring as its work, and to stimulate others to investigate as patiently and earnestly the varied phenomena whose basis is "molecular mobility."

Sir William Thomson, in proposing a vote of thanks to the lecturer, called attention to a diagram on the wall recording the rates of passage of gases by diffusion, effusion, transpiration, and by the peculiar passage through such "colloid septa" as non-crystalline metals or india-rubber; and he stated that before Graham's time these valuable physical constants were absolutely unknown. They had listened with much interest to the connection which had been traced between Graham's law of diffusion and the science of molecular physics, as well as to the account of Graham's work generally, so carefully set before them by Graham's pupil and friend.

PRELIMINARY NOTE ON THE SUBSTANCES WHICH PRODUCE THE CHROMOSPHERIC LINES ${ }^{1}$

IITHERTO, when observations have been made of 1 the lines visible in the sun's chromosphere, by means of the method introduced by Janssen and myself in 1868 , the idea has been that we witness in solar storms the ejection of vapours of metallic elements with which we are familiar from the photosphere.

A: preliminary discussion of the vast store of observations recorded by the Italian astronomers (chief among them Prof. Tacchini), Prof. Young, and myself, has shown me that this view is in all probability unsound. The lines observed are in almost all cases what I have elsewhere termed and described as basic lines; of these I only need for the present refer to the following :-

$b_{3}$ ascribed by Ångström and Kirchhoff to iron and nickel.

$b_{4} \quad \because \quad$ Angströin to magnesium and iron.

5268 by "Angström to cobalt and iron.

5269 ,", calcium and iron.

5235 " cobalt and iron.

5017 ", nickel.

$4215 \quad$ ", ," $\quad$ calcium, but to strontium by myself. 5416 an unnaned line.

Hence, following out the reasoning employed in my previous paper, the bright lines in the solar chromosphere are chiefly lines due to the not yet isolated bases of the so-called elements, and the solar phenomena in their totality are in all probability due to dissociation at the photospheric level, and association at higher levels. In this way the vertical currents in the solar atmosphere, both ascending and descending, intense absorption in sun-spots, their association with the faculæ, and the apparently continuous spectrum of the corona and its structure, find an easy solution.

We are yet as far as ever from a demonstration of the cause of the variation in the temperature of the sun; but the excess of so-called calcium with minimum sunspots, and excess of so-called hydrogen with maximum sun-spots follow naturally from the hypothesis, and afford indications that the temperature of the hottest region in the sun closely approximates to that of the reversing layer in stars of the type of Sirius and $a$ Lyræ.

If it be conceded that the existence of these lines in the chromosphere indicates the existence of basic molecules in the sun, it follows that as these lines are also ' Paper read at the Royal Society on January 23, by J. Norman Lockyer, seen generally in the spectra of two different metals in the electric arc, we must be dealing with the bases in the arc also.

\section{ON A THEORY OF THE VISCOSITY OF THE EARTH'S MASS ${ }^{1}$}

$\mathrm{T}$ these two papers the investigation is continued of the physical results which follow from the theory that the mass of the earth is either viscous or imperfectly elastic. In the first paper of the series (which was read before the Royal Society on May 23, I878, and of which an account appeared in NATURE, vol. xviii. p. 265 ) the theory of the bodily tides of such a spheroid was considered. In that paper it was shown that the bodily tides would lag, and that this lagging would produce an acceleration of the time of high water of the oceanic tides relatively to the nucleus. The author's attention was directed to the tidal reports of the British Association by Sir W. Thomson, and he has tried to find whether the tidal observations give any indications of a yielding of the earth's mass. The theory of the semi-diurnal and diurnal oceanic tides is so imperfect that it is impossible to say whether or not high water takes place earlier than it would do on a rigid nucleus; the long-period tides are those from which alone any indications are to be expected.

The fortnightly tide is the most marked of these, but its height is very small, and the results in the tidal observations show so much irregularity that it cannot be asserted with certainty that they represent the true fortnightly tide. Nevertheless, it is interesting to learn that, out of eleven years of observation at Ramsgate, Liverpool, Hartlepool, Brest, and Kurrachee, the fortnightly tide appears to be accelerated in eight cases and only retarded in three. Although the accelerations are exceedingly irregular, it may perhaps be maintained that these observations give some indications of a tidal yielding of the earth's mass.

The first of the two papers of which we are here speaking deals with the effects of the tidal distortion of the spheroid on its rotation, and with the reaction on the tide-raising satellite. An account of some of the results of the investigation was read before the British Association at Dublin, and an abstract appeared in NATURE, vol. xviii. p. 580, and therefore the principal results will be here merely repeated.

For convenience of diction the spheroid is spoken of as the earth and the tide-raising body as the moon.

It was found, then, that the obliquity of the ecliptic, the length of day and of the month, become variable, and that, if we look into the remote past, we find the obliquity less, and the day and month very much shorter than at present. When the changes were traced backwards as far as possible it was found that the whole diminution in the obliquity was about $10^{\circ}$, and that the beginning from which the earth and moon must have started was a state in which they rotated, as though fixed rigidly together, in $5 \mathrm{~h}$. $4 \mathrm{Om}$, the moon being then only Io,000 miles distant from the earth's centre.

In the second paper (read before the Royal Society on December 19) some other problems were considered. The first of these is concerning the secular distortion of the spheroid. Under the attraction of the moon the earth becomes distorted into an ellipsoidal shape, with the longest axis in the plane of the equator, but, since the tide lags, this longest axis does not point directly towards the moon. The excess of the attraction of the moon on the nearer protuberance above that on the further one gives rise to the tidal frictional couple. This couple tends to retard the earth's rotation; but it is clear that unless the tidal protuberance has some special form

I An account of two papers, "On the Precession of a Viscous Spheroid, and on the Remote History of the Earth," and "Problems Connected with the Tides of a Viscous Spheroid," by G. H. Darinin, read before the Royal Society on December 19,1878 . 
the whole earth cannot be retarded exactly as though it were a rigid body. Now the tidal protuberance has not this required form, and therefore there results a slow secular distortion of the earth arising from the unequal distribution over the surface of the forces which constitute the tidal frictional couple.

The greater part of the pull which retards the rotation is applied in the equatorial regions, and therefore the rotation of those regions will be more rapidly retarded than that of the polar regions. As the earth's rotation is from west to east, it follows that the polar regions will outstrip the equator and will move very slowly from west to east relatively to the equatorial parts.

The exact mathematical solution for this kind of a distortion of a viscous spheroid shows that it consists in a simple cylindrical motion round the axis of rotation, each point moving from east to west with a linear velocity proportional to the cube of its distance from that axis.

The distortion of the surface of the globe consists of a motion in longitude from west to east, relatively to a point in the equator, the rate of change of longitude being proportional to the square of the sine of the latitude.

Numerical calculation shows, however, that in the later stages of the earth's history (the development being supposed to follow the laws found in the paper on "Precession") the distortion must have been very small. With a certain assumed viscosity it is found that, looking back $45,000,000$ years, a point in latitude $60^{\circ}$ would lie $14^{\prime}$ further east than at present. From this it follows that this cause can have had little or nothing to do with the crumpling of geological strata.

As, however, the distorting force varies inversely as the sixth power of the moon's distance, it seems possible that in the very earliest stages this cause may have had sensible effects. It is therefore noteworthy that the wrinkles raised on the surface would run north and south in the equatorial regions, with a tendency towards northeast and south-west in the northern hemisphere, and north-west and south-east in the southern one. The intensity of the distorting force at the surface varies as the square of the cosine of the latitude.

An inspection of a map of the earth shows that the continents (or large wrinkles) conform more or less to this law. But Prof. Schiaparelli's map of Mars ${ }^{1}$ is more striking than that of the earth, when viewed by the light of this theory; but there are some objections to its application to the case of Mars. If, however, there is any truth in this, then it must be postulated, that after the wrinkles were formed the crust attained sufficient local rigidity to resist the obliteration of the wrinkles, whilst the mean figure of the earth adjusted itself to the ellipticity appropriate to the slackening diurnal rotation: also, it must be supposed that the general direction of the existing continents has lasted through geological history.

The second problem considered in this paper is concerning the distribution of the heat, which would be generated by the internal friction of the tidal distortion.

It was shown in the preceding paper that a very large amount of heat might be thus generated, and it appeared at first sight as though this might serve to explain in part the observed increase of underground temperature; but the solution of a certain problem concerning the cooling of an infinite slab of rock 8,000 miles thick, in which heat is being generated according to a certain law of distribution, shows that the frictional heat could not possibly explain a rate of increase of underground temperature near the earth's surface of more than $I^{\circ}$ Fahr. in 2,600 feet.

It follows, therefore, that Sir W. Thomson's investigation of the secular cooling of the earth cannot be sensibly affected by this cause.

${ }^{2}$ Memorie della Società degli Spettroscopisti Italiani, 1878 , vol. vii.
The last part of the paper does not lead to results of interest to the general reader, as it is concerned with the part played by inertia in the tides of viscous, fluid, and elastic spheres.

\section{INDIAN METEOROLOGY"}

$\mathrm{TN}$ the article "Atmosphere" of the Encyclopadia Britannica it has been justly remarked that one of the most important steps that could be taken towards the development of the science of meteorology would be extensive series of observations from such countries as India, which offers splendid contrasts of climate at all seasons; has a surface covered at one place with the richest vegetation, and at others with vast stretches of sandy deserts, and presents extensive plateaus and sharp ascending peaks, all which conditions are indispensable for collecting the data required for the solution of the problem of atmospheric physics. In working out this problem it is necessary, owing to its extreme complexity and difficulty, to give attention, not merely to questions immediately bearing on the physics of the atmosphere, but also to climatic and other practical inquiries, which may be handled with comparative ease and which afford results that contribute indirectly but very materially to the solution of the higher problem. The publications enumerated below admirably follow up this two-fold line of inquiry, and even already several important practical and theoretical conclusions seem not far from the point of being reached by the meteorologists of India.

The "Report on the Meteorology of India" is the second Annual Report issued since the administration of the Indian Meteorological Establishment was concentrated in the Central Office at Calcutta for the whole of India including British Burmah and the Islands of the Bay. In the scheme of publication of the monthly results of the observations made at the various stations over India, we note with satisfaction that the form proposed by the Permanent Committee of the Meteorological Congress at Vienna has not been adopted in some of its more important details. Thus in Mr. Blanford's tables, instead of a general monthly mean of atmospheric pressure, the mean monthly pressure for each hour of observation is given -an essential requisite for the presentation of the data required in discussing various of the more important problems of international meteorology. Indeed these tables possess the very high merit of being, with perhaps one exception, entirely suited for the discussion of climatic questions of an international character-the single exception being the lumping together of the two or four daily observations of the winds into one monthly mean, instead of a monthly mean for each hour of observation as is so admirably carried out by Professor Rubenson in his annual reports of Swedish meteorologry.

The most interesting part of this report is that which deals with the failure of the rains in Western and Southern India which resulted, as is only too well known, in one of the most terrible and wide-spread famines of recent years. The mode of treatment is grounded on the practice adopted by the Office, in framing forecasts of coming seasons to which we have several times drawn the attention of the readers of NATURE (vol xiii. p. 66, \&c.), and which may be described as proceeding on the assumption that there is a certain persistency in meteorological conditions; that, for instance, the longer a given state of weather has lasted, the less the probability of a speedy change ; and that as regards the distributions of pressure, on which weather is so dependent, certain states of the atmosphere tend to perpetuate or reproduce themselves in the same region in such a manner as to maintain a

" "Report on the Meteorology of India in 1876 ," By Henry F. Blanford. "The Indian Meteorologist's Vade-Mecum." By Henry F. Blanford. "Indian Meteorological A emoirs;" issued under the d rection of Henry $F$. "Indian Meteorological Memoirs;" issued under the d "ection of Henry F. dency." By Charles Chambers, F.R.S. 